\title{
Interactive comment on "Modelling the residual mean meridional circulation at different stages of stratospheric warming events" by Andrey V. Koval et al.
}

\section{Anonymous Referee \#1}

Received and published: 11 November 2020

The paper reports on the study of the residual mean circulation during sudden stratospheric warmings (SSW) using an ensemble of simulations with the mechanistic general circulation model. The use of 19 simulations helps to separate essential changes caused by SSW from peculiarities of particular events. The diagnostics was extended to residual fluxes of ozone. I find the results solid, interesting and deserving publication. There were, however, some questions and comments, while reading the manuscript. The authors may wish to address them in what I expect to be a minor revision. They are listed below in the sequential order.

Line 50. SSW, major and minor, do also occur in the Southern hemisphere, e.g., 
L. 64. "studied for the first time". Further in the text, authors cite other papers on the same subject, so this work is not the first of its kind. May be the "first time with this model"?

L. 93. How the normal modes were included? By adding additional disturbances at the lower boundary? Or they were generated by the model in response to daily variations of the solar flux?

L. 95-96. That is, at 50, but not $30 \mathrm{~km}$ ? This is unclear from the text. Formulas (3) and (4). For me they look precisely equal to (1) and (2). Are they just another form of the latter, or am I missing something?

L. 206. "Increased downward ... velocities". As follows from Fig. 2b, the downward velocities between 20 and $60 \mathrm{~km}$ weaken during SSW. Is it a typo?

L. 208. Again, if the downward velocities are weaker over high latitudes, they cannot help with heating the atmosphere.

L. 214. "Parcels" rather than "particles" is more suitable word for gas elements.

L. 216. " For short time intervals". Here it would be appropriate to give the characteristic chemical lifetime of ozone at these heights in comparison with 11-day intervals of interest.

L. 217. Specify what data the MUAM ozone model is based on.

L. 214. Is that really "monthly-mean" ozone? How good is to use monthly means for such rapid events as SSW? Or some sort of time interpolation was used?

L. 245. Ozone fluxes enhance the residual circulation? They just reflect it. A rewording is needed.

L. 248-250. I would recommend to move reservations concerning the fixed ozone 
distribution to the beginning of the section. Readers would then understand from the beginning that this is just a rough estimate.

ANGEOD

L. 266. Aren't the fluxes decrease at this location, as Fig. $2 b$ shows?

Interactive comment on Ann. Geophys. Discuss., https://doi.org/10.5194/angeo-2020-71, 2020.

Interactive

comment 